\title{
Evaluation of kidney dysfunction and angiotensinogen as an early novel biomarker of intrauterine growth restricted offspring rats
}

\author{
Yayoi Murano', Naoto Nishizaki², Amane Endo², Naho Ikeda', Tomonosuke Someya ${ }^{2}$, Mayu Nakagawa', Taichi Hara', \\ Koji Sakuraya ${ }^{3}$, Satoshi Hara ${ }^{2}$, Daishi Hirano ${ }^{4}$, Mitsuyoshi Suzuki', Hiromichi Shoji', Shuichiro Fujinaga ${ }^{4}$, Yoshiyuki Ohtomo ${ }^{2}$ \\ and Toshiaki Shimizu'
}

BACKGROUND: Few studies have addressed the growing concerns of chronic kidney diseases in children with intrauterine growth restriction (IUGR). Therefore, the purpose of this study was to evaluate long-term kidney dysfunction and determine if urinary angiotensinogen (AGT) was suitable as a novel early biomarker for kidney dysfunction in IUGR offspring. METHODS: Pregnant rats underwent bilateral uterine artery ligation, and as a control group, sham surgeries were performed.

RESULTS: The birth weight was reduced, the urinary AGT to creatinine ratio was significantly higher at week 20, and urinary protein levels were significantly higher at week 32 in IUGR rats than in control rats. On the other hand, the histological findings at week 32 revealed long-term kidney dysfunction, more severe glomerulosclerosis, and greater glomerular diameters in IUGR rats. Moreover, AGT mRNA expression and immunohistological staining were significantly increased in IUGR rats; this suggests that the intrarenal renin-angiotensin system (RAS) contributes to renal dysfunction of IUGR offspring.

CONCLUSION: Urinary AGT elevation prior to urinary protein levels suggests that AGT is an early biomarker. At week 32, kidney dysfunction was severe in IUGR rats and intrarenal RAS appeared to be one of the causes.

$\mathbf{S}_{\mathrm{n}}^{\mathrm{i}}$ ince Barker et al. (1) first proposed that many adult diseases may have origins in fetal life, subsequent studies have clarified the associations between intrauterine growth restriction (IUGR) and obesity, hypertension, insulin resistance, and coronary artery disease. Luyckx and Brenner (2) applied these findings to the development of chronic kidney disease and following studies were conducted in animal models as well as human subjects. In these animal studies, IUGR was typically found to be induced by maternal food restriction (3-5); however, no applications to current IUGR rates in developed countries were made; malnutrition during pregnancy due to food restriction during pregnancy is no longer the main cause of IUGR in developed countries.
Most cases of IUGR reportedly result from decreased placenta blood flow (6). A decrease in placenta blood flow by maternal bilateral uterine artery ligation was shown in an animal model; however, relatively few studies have been conducted to evaluate subsequent kidney dysfunction. To the best of our knowledge, no previous study has assessed the long-term effects of IUGR on kidney function. Therefore, in this study, we examined kidney dysfunction in a rat model of IUGR induced by bilateral uterine artery ligation for $32 \mathrm{wk}$ after birth.

On conducting this study, we also decided to identify clues to detect dormant chronic kidney disease in adults who were born with a low birth weight. Owing to developments in the field of neonatology, the survival rate of infants with a low birth weight has continued to increase (7). Although the first generation of infants from the "surfactant era" who may have chronic kidney disease pathogenesis are now reaching young adulthood, no reliable screening methods or followup procedures to monitor these dominant diseases have been established to date (8). Recently, urinary angiotensinogen (AGT) was proposed as a novel biomarker to detect kidney dysfunction caused by renal diseases, such as obstructive nephropathy (9), diabetic nephropathy $(10,11)$, membranous nephropathy, chronic glomerulonephritis, and immunoglobulin A nephritis $(12,13)$. However, few studies have focused on urinary AGT in patients born as low-birth-weight infants, and a literature search retrieved no related studies using animal models.

We hypothesized that urinary AGT in IUGR rats arises prior to the increase in urinary protein excretion. Therefore, in this study, we determined whether urinary AGT could serve as an early marker of kidney dysfunction in IUGR offspring of rats.

\section{RESULTS}

Birth Weight, Body Weight, and Kidney Weight

Birth weight was significantly lower in the IUGR group than in the control group $(6.55 \pm 0.16$ vs. $7.82 \pm 0.07 \mathrm{~g} ; P=0.001)$. However, at $32 \mathrm{wk}$ of age, there were no significant differences 
Table 1. Comparison of body weight, body length, kidney weight, and kidney length

\begin{tabular}{lccc}
\hline & $\begin{array}{c}\text { IUGR } \\
(n=10)\end{array}$ & $\begin{array}{c}\text { Control } \\
(n=10)\end{array}$ & $P$ \\
\hline Birth weight (g) & $6.55 \pm 0.16$ & $7.82 \pm 0.07$ & $0.001^{*}$ \\
Body weight at 32 wk (g) & $633.2 \pm 7.3$ & $633.4 \pm 3.6$ & 0.34 \\
Body length at 32 wk (cm) & $27.0 \pm 0.1$ & $26.3 \pm 0.1$ & 0.16 \\
Kidney weight/body weight (\%) & $0.28 \pm 0.0$ & $0.28 \pm 0.0$ & 0.35 \\
Kidney length/body length (\%) & $0.08 \pm 0.0$ & $0.08 \pm 0.0$ & 0.45 \\
\hline
\end{tabular}

Data are presented as mean $\pm \mathrm{SEM} ; n=10$ per group. Birth weight was significantly lower in the IUGR group. There were no significant differences in body weight, body length, kidney weight to body weight ratio, and kidney length to body length ratio between groups.

IUGR, intrauterine growth restriction.

${ }^{*} P<0.05$.

Table 2. Comparison of blood parameters between IUGR and control groups

\begin{tabular}{lccc}
\hline & IUGR $(n=10)$ & Control $(n=10)$ & $P$ \\
\hline BUN $(\mathrm{mg} / \mathrm{dl})$ & $19.2 \pm 0.3$ & $21.0 \pm 0.2$ & 0.11 \\
Creatinine $(\mathrm{mg} / \mathrm{dl})$ & $0.35 \pm 0.0$ & $0.38 \pm 0.0$ & 0.11 \\
AGT $(\mathrm{mg} / \mathrm{ml})$ & $1.15 \pm 0.0$ & $1.28 \pm 0.0$ & 0.18
\end{tabular}

Data are presented as mean $\pm S E M ; n=10$ per group. Existing markers of kidney dysfunction: there were no significant differences in BUN or creatinine levels between groups. Serum AGT, which affects the systemic RAS, did not differ between groups. $A G T$, angiotensinogen; BUN, blood urea nitrogen; IUGR, intrauterine growth restriction.

in body weight between groups $(633.2 \pm 7.3$ vs. $633.4 \pm 3.6 \mathrm{~g} ; P$ $=0.34$ ). After adjusting kidney weight to body weight, there were no significant differences between groups $(0.28 \pm 0.0$ vs. $0.28 \pm 0.0 \% ; P=0.35$; Table 1 ).

\section{Body Length and Kidney Length}

At $32 \mathrm{wk}$ of age, there were no significant differences in body length between the IUGR and control groups $(27.0 \pm 0.1$ vs. $26.3 \pm 0.1 \mathrm{~cm} ; P=0.16)$. After adjusting for body length, there were no significant differences in kidney length between groups ( $0.08 \pm 0.0$ vs. $0.08 \pm 0.0 \% ; P=0.45$; Table 1 ).

\section{Blood Analysis}

Analysis of the blood samples obtained at $32 \mathrm{wk}$ of age showed no significant differences in blood urea nitrogen, creatinine, or AGT levels between the IUGR and control groups (blood urea nitrogen: $19.2 \pm 0.3$ vs. $21.0 \pm 0.2 \mathrm{mg} / \mathrm{dl} ; P=0.11$; creatinine: $0.35 \pm 0.0$ vs. $0.38 \pm 0.0 \mathrm{mg} / \mathrm{dl} ; P=0.11$; and AGT: $1.15 \pm 0.0$ vs. $1.28 \pm 0.0 \mathrm{mg} / \mathrm{l} ; P=0.18$; Table 2).

\section{Urine Analysis}

At 4 wks of age, there were no significant differences in the ratio of urinary protein and AGT to creatinine between groups $(0.40 \pm 0.05$ vs. $0.45 \pm 0.02 \mathrm{~g} / \mathrm{g} \mathrm{Cr} ; \mathrm{P}=0.19$; and $0.00 \pm 0.00$ vs. $0.009 \pm 0.00 \mathrm{mg} / \mathrm{g} \mathrm{Cr}, P=0.18)$. At $20 \mathrm{wk}$ of age, there were also no significant differences between the ratio of urinary protein to creatinine between groups $(2.32 \pm 0.13$ vs. $1.30 \pm 1.19 \mathrm{~g} / \mathrm{g} \mathrm{Cr}$; $P=0.11$ ), while the ratio of urinary AGT to creatinine in the IUGR group was significantly higher than that in the control
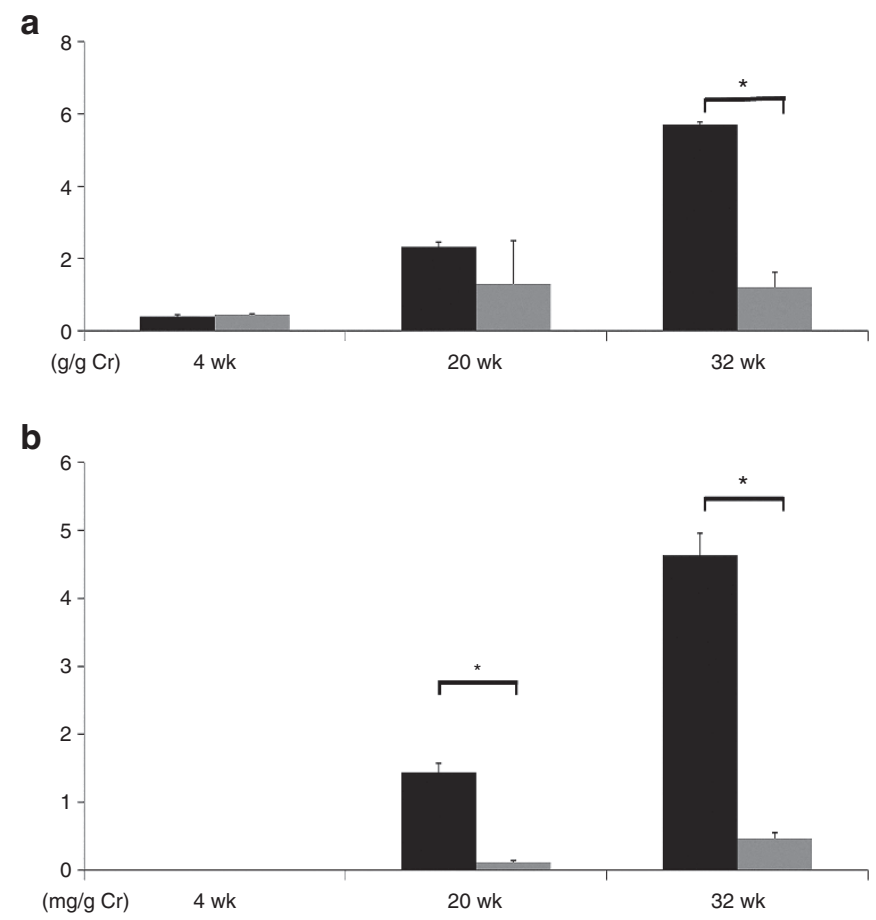

Figure 1. Urine analysis. (a) Comparison of urinary protein/creatinine ratio and (b) urinary AGT/creatinine ratio. Results expressed as mean \pm SEM. $n=10$ per group. ${ }^{*} P<0.05$. At week 4 , there were no significant differences in urinary AGT/creatinine ratio or urinary protein/creatinine ratio between groups. At week 20, urinary AGT/creatinine ratio was significantly higher in the IUGR group, prior to the increase in the urinary protein/creatinine ratio. At week 32, there were no significant differences in the urinary $\mathrm{AGT} / \mathrm{creatinine}$ ratio or urinary protein/creatinine ratio between groups. Dark grey shade indicates IUGR group and light gray shade indicates control group. AGT, angiotensinogen; IUGR, intrauterine growth restriction.
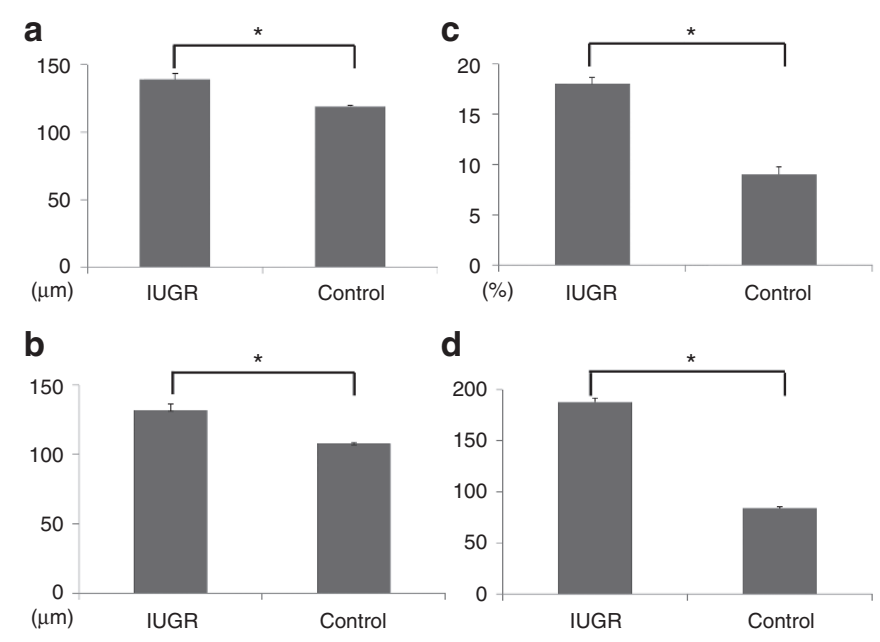

Figure 2. Kidney morphology. (a) mean glomerular diameter of 100 glomeruli and (b) the estimated radius from the MPA method. (c) Maximum planar area of glomeruli (PA ${ }_{\max }$ ) and (d) Sclerotic index (GSI) at week 32 between the IUGR and control groups. Results expressed as mean \pm SEM. $n=10$ per group ${ }^{*} P<0.05$. There were significant differences in the mean diameter of glomeruli, $\mathrm{PA}_{\max }$ and GSI between groups. GSI, glomerulosclerosis index; IUGR, intrauterine growth restriction. 
a

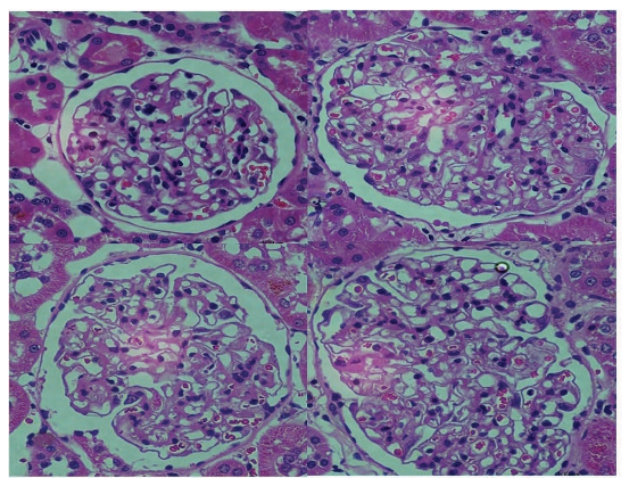

c

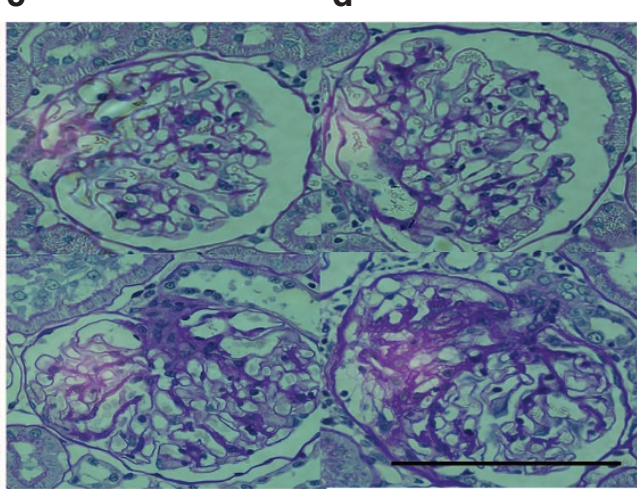

Figure 3. Light microscopy of glomeruli at week 32 (hematoxylin-eosin (HE) and PAS staining of 3- $\mu$ m-thick sections, $\times 400$ ). (a) Glomeruli of control rats, HE stained. (b) Glomeruli of IUGR rats, HE stained. (c) Glomeruli of control rats, PAS stained. (d) Glomeruli of IUGR rats, PAS stained. In control group, the glomerular lesions appeared almost normal, and the diameters were wider in IUGR rats. Sclerotic glomerular lesions were more extensive in the IUGR rats. Bar $=100 \mu \mathrm{m}$. PAS, periodic acid-Schiff. IUGR, intrauterine growth restriction.

a

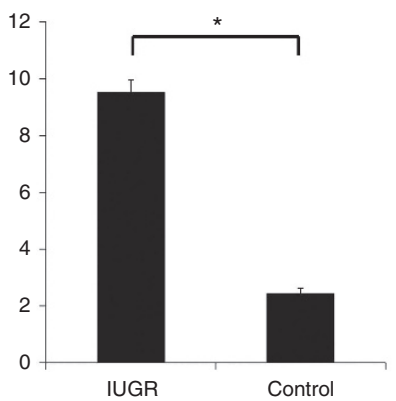

b

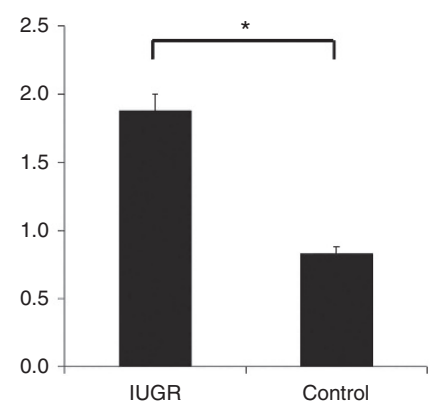

Figure 4. Expression of intrarenal AGT. (a) The percentage of area expressing AGT protein and (b) The expression of AGT mRNA. Results are expressed as mean \pm SEM. $n=10$ per group. ${ }^{*} P<0.05$. The mean percentage of area expressing AGT of 10 fields per rat was calculated. In IUGR group, the extent of the AGT-positive area and the expression of AGT mRNA were significantly greater. AGT, angiotensinogen; IUGR, intrauterine growth restriction.

group ( $1.44 \pm 0.13$ vs. $0.12 \pm 0.02 \mathrm{mg} / \mathrm{g} \mathrm{Cr} ; P=0.001)$. At $32 \mathrm{wk}$ of age, the ratios of urinary protein and AGT to creatinine were significantly higher in the IUGR group than those in the control group (5.70 \pm 0.41 vs. $1.21 \pm 0.08 \mathrm{~g} / \mathrm{g} \mathrm{Cr} ; P<0.004$ and $4.63 \pm 0.33$ vs. $0.47 \pm 0.08 \mathrm{mg} / \mathrm{g} \mathrm{Cr} ; P=0.004$; Figure 1 ).

\section{Kidney Morphology}

The average diameter of 100 glomeruli, as determined by hematoxylin-eosin staining, was significantly greater in the IUGR group than that in the control group $(139 \pm 4.4$ vs. $119 \pm 0.7 \mu \mathrm{m} ; P=0.007)$. The estimated radius from the maximal profile area method and the maximum planar area of glomeruri were both significantly greater in the IUGR group than that in the control group $(132 \pm 2.1$ vs. $108 \pm 1.4 \mu \mathrm{m} ; P=0.006$ and $18 \pm 0.64$ vs. $9 \pm 0.78 \%, P=0.01$ ). The glomerulosclerosis index of 100 glomeruli examined by PAS staining was also significantly greater in the IUGR group than that in the control group (188 \pm 3.7 vs. $84 \pm 1.4 ; P<0.001$; Figure 2). In Figure 3, glomeruli pictures which are larger and more sclerotic in IUGR are shown. However, there were no significant differences in the extent of interstitial fibrosis in the renal cortex, as

examined by Masson's trichrome staining, between two groups $(11.4 \pm 0.69$ and $8.8 \pm 0.48 \% ; P=0.89)$.

\section{Expression of Intrarenal AGT}

Immunohistochemical analysis of AGT. The average percentage of areas exhibiting AGT protein expression in the kidney cortex was significantly higher in the IUGR group than that in the control group ( $9.52 \pm 0.44$ vs. $2.45 \pm 0.17 \% ; P=0.003$; Figure 4).

Real-time PCR of AGT. AGT mRNA expression levels in the kidney cortex were significantly higher in the IUGR group than that in the control group $(1.88 \pm 0.12$ vs. $0.83 \pm 0.05$, relative ratio to one randomly selected control rat; $P=0.007$; Figure 4).

\section{DISCUSSION}

Here, we found that offspring rats with IUGR, which was induced by the ligation of the maternal bilateral uterine arteries, developed kidney dysfunction by the age of $32 \mathrm{wk}$. Furthermore, we showed that urinary AGT levels were increased in the IUGR model prior to the elevation of urinary protein excretion, suggesting that urinary AGT is suitable as a biomarker of kidney dysfunction.

There were significant differences in birth weight between the IUGR and control groups. However, at $32 \mathrm{wk}$ of age, there were no significant differences in body weight, kidney weight, body length, or relative body length between groups. Although several studies have reported that body weight and relative kidney weight were significantly lower in the IUGR group than those in the control group $(4,5)$, our results were not in agreement. However, the animals were killed at younger ages in these previous studies. The results of the only study of IUGR in a rat model at $32 \mathrm{wk}$ of age were similar to our findings (3).

Here, we investigated the usefulness of urinary AGT as an early biomarker of kidney dysfunction in IUGR offspring. There have been only a few human studies that evaluated the relationship between AGT and kidney dysfunction in IUGR (14); to the best of our knowledge, there has been no such study to date using an animal model. Therefore, this study is the 
first to examine urinary AGT in IUGR offspring in an animal model. Our results showed that urinary AGT levels were significantly higher in the IUGR group than that in the control group at $20 \mathrm{wk}$ of age, which is regarded as adolescence in humans. At $32 \mathrm{wk}$ of age, which is equivalent to adulthood in humans, both urinary AGT and protein levels were significantly higher in the IUGR group than that in the control group. It should be noted that urinary AGT increased prior to the onset of proteinuria or elevations of serum creatinine or blood urea nitrogen levels, which is usually clinically referenced. The results of this study indicated that urinary AGT level increased prior to any other marker including urinary protein. Furthermore, the fact that there were no differences in serum AGT levels between groups suggests that this increase in urinary AGT was not caused by overflow of blood but by intrarenal injury.

Although the mechanism of renal dysfunction in IUGR offspring has not been completely clarified, substantial evidence indicates that the renin-angiotensin system (RAS) may be a possible pathway (15). In many other diseases including obstructive nephropathy (9), diabetic nephropathy (15), membranous nephropathy, chronic glomerulonephritis, and IgA nephritis $(11,12)$, the activation of the intrarenal RAS plays important role in kidney dysfunction. In IUGR, there exists an immature function of RAS (14), which may result in inappropriate activation of RAS. AGT is the only known substrate for renin that is a rate-limiting enzyme of the RAS and is enhanced in the progression of glomerular disease (16). In addition, AGT is known to enhance intrarenal RAS activity, which has recently attracted attention. Among these new trends in studies of renal function, examining AGT in IUGR offspring has gained acceptance. Moreover, we confirmed that both AGT protein and mRNA expression levels were increased in IUGR. These findings support the theory that the RAS, particularly the intrarenal RAS, contributes to kidney dysfunction in IUGR. In IgA nephropathy, blockage of RAS is successful in its mitigation and therapy of it (17). Considering the fact that RAS plays an important role in IUGR, RAS blockage would prevent kidney dysfunction in IUGR patients.

Histological analysis also indicated long-term kidney damage among rats in the IUGR group, as urinary AGT levels increased in this group than in the control group. One method to evaluate glomerulosclerosis is to score each glomerulus according to the extent of sclerotic injury, as first reported by Fujihara et al. (18) and subsequently referenced by several other studies $(19,20)$. In agreement with the findings of this study, those of previous studies reported that focal segmental glomerulosclerosis was a cause of renal dysfunction in the IUGR offspring (21).

The larger glomerular diameters in the IUGR group than the control group can be explained by the hypothesis proposed by Brenner et al. (22), which states that congenital deficits in nephron number, occurring in IUGR offspring, result in decreased filtration surface area and increased renal blood flow per glomerulus in an attempt to maintain a normal glomerular filtration rate, resulting in glomerulomegaly (Brenner's hyper filtration hypothesis). To the best of our knowledge, this is the first study to assess the relationship between glomerular diameter and renal function in an animal model of IUGR.

There were no significant differences in the extent of interstitial fibrosis in the renal cortex, as examined by Masson's trichrome staining. This finding supports the proposition that the extent of glomerular injury was an indicator of kidney injury in IUGR. Urushihara and Kobori (16) reported that AGT expression was enhanced by the progression of glomerular disease, which also supports our hypothesis.

The findings described above led us to conclude that elevated urinary AGT is suitable as a new biomarker of dormant chronic kidney disease to predict future proteinuria and kidney injury. There were three limitations to this study that should be addressed. First, we did not evaluate histological kidney injury at week 20, when urinary AGT levels increased, while the other markers did not. Second, we could not quantify the total number of glomeruli, which is important to validate Brenner's hypothesis. Third, this study compared only two groups, and no cutoff levels were established.

In this study, we prepared IUGR offspring rats by bilateral uterine artery ligation of the maternal rats and for the first time showed that renal dysfunction occurred in these offspring during maturation by comparison with offspring rats in the control group. In addition, we clarified that the measurement of urinary AGT levels, which reflects hyperfunction of the intrarenal RAS, presents a new and effective marker to predict future renal dysfunction.

\section{METHODS}

\section{Animals}

Female Sprague Dawley rats at gestational day 14 were purchased from Nihon SLC (Shizuoka, Japan) and housed in individual cages in the same room at $24-25{ }^{\circ} \mathrm{C}$ and relative humidity of $60 \%$ under a 12:12-h light-dark cycle with free access to food and water at the Juntendo University Animal Care Facility (Tokyo, Japan). Each rat was anesthetized by isoflurane inhalation on gestational day 18 . The rats were divided into two groups: one that underwent bilateral uterine artery ligation with 3-0 silk and a second that underwent sham surgery. The male offspring of artery ligated rats were included in the IUGR group and the rats that underwent sham surgery were included in the control group. The study protocol was approved by the Animal Care Committee of Juntendo University.

Urine was collected from the experimental rats on 4,20, and $32 \mathrm{wk}$ of birth to examine urinary protein, creatinine, and AGT levels. All rats were terminated at wk 32 , during which blood samples were collected and the kidneys were excised and weighed.

\section{Blood Analysis}

Serum was obtained from all whole-blood samples by centrifugation at $3,000 \mathrm{rpm}$ for $20 \mathrm{~min}$ at $4{ }^{\circ} \mathrm{C}$ and stored at $-20^{\circ} \mathrm{C}$ until analyzed. Blood urea nitrogen and creatinine levels were measured using an automatic analyzer (JCA-BM8060; Beckman Coulter, Fulleton, CA). Serum AGT levels were measured using a commercial enzyme-linked immunosorbent assay kit (Immuno-Biological Laboratories, Gumma, Japan).

\section{Urine Analysis}

The rats were placed in individual cages for $24 \mathrm{~h}$ to collect urine samples, which were centrifuged at $3,000 \mathrm{rpm}$ for $10 \mathrm{~min}$ at $4{ }^{\circ} \mathrm{C}$. Absorbance of urinary creatinine was measured by enzymatic methods using an automatic analyzer (Olympus AU 5431; Beckman Coulter,) at $545 \mathrm{~nm}$. Urine protein levels were measured by the Pyrogallol red method using an automatic analyzer (Hitachi 7170; Hitachi, Tokyo, Japan). Urinary AGT levels were measured using a commercial enzymelinked immunosorbent assay kits (Immuno-Biological Laboratories). 


\section{Kidney Morphology}

Tissue samples were fixed in $10 \%$ formalin, embedded in paraffin, and cut into sections stained with hematoxylin-eosin, periodic acid-Schiff, and Masson's trichrome reagents. Morphometric evaluations were performed by a single observer who was blinded to all study variables. The diameters of 100 glomeruli in hematoxylin-eosin-stained sections were measured for each rat. In addition, we evaluated the estimated diameters of 20 glomeruli by maximal profile area volumes mentioned before by Lane et al. to confirm this result. Maximum planar area of glomeruli was calculated from 20 glomeruli. KS 400 (Carl Zeiss Imaging Solutions, Hallbergmoss, Germany) was used to estimate these subjects $(23,24)$. The severity of glomerulosclerosis was evaluated by assigning a score to each glomerulus according to the extent of sclerotic injury in periodic acid-Schiff-stained sections as described in previous reports $(18,19): 0$ $=$ intact glomeruli; $1=$ lesions affecting $<20 \%$ of the glomerular area; 2 $=$ lesions affecting $21-40 \%$ of the glomerular area; $3=$ lesions affecting $41-60 \%$ or less of the glomerular area; $4=$ lesions affecting $61-80 \%$ of the glomerular area; and $5=$ lesions affecting $>80 \%$ of the glomerular area. The glomerulosclerosis index was calculated by examining 100 glomeruli from each rat. Interstitial fibrosis was evaluated by blue staining with Masson's trichrome. A color image analyzer (Image J 1.46 for Windows; National Institutes of Health, Bethesda, MD) was used for quantification of the percentage of injured areas.

\section{Immunohistochemical Staining for AGT Measurements}

AGT protein levels in the kidneys were assessed by immunohistochemical staining. Renal sections were fixed in $10 \%$ (v/v) formalin, embedded in paraffin, and sliced into 3 - $\mu \mathrm{m}$-thick sections. After deparaffinization in xylene, antigens were retrieved in citrate buffer $\left(\mathrm{pH} \mathrm{6.0)}\right.$ at $121{ }^{\circ} \mathrm{C}$ for $10 \mathrm{~min}$. Then, the sections were incubated with primary antibodies against rat AGT (Immuno-Biological Laboratories). Subsequently, the sections were incubated with peroxidase micropolymer linked to antirabbit IgG antibody using the ImmPRESS polymerized reporter enzyme staining system (Vector Laboratories, Burlingame, CA). Development was performed with 3,3'-diaminobenzidine tetrahydrochloride (Wako Pure Chemical Industries, Osaka, Japan). Samples were also counterstained with hematoxylin before analysis. For each microscopic field, the AGT-positive area (stained brown) was automatically calculated using the above-mentioned software. The affected area was divided by the total area of the microscopic field. Ten random microscopic fields were examined for each rat, and the percentages of positive lesions were averaged.

\section{Real-Time PCR for AGT}

RT-PCR was performed to detect expression of AGT in the renal cortex. TaqMan probe-based quantitative RT-PCR was performed using cDNA synthesized from kidney biopsy RNA preparations (ReverTraAce qPCR RT Master Mix; Toyobo, Osaka, Japan) and analyzed using a 7500 Fast Real-Time PCR system (Applied Biosystems, Foster City, CA) with default settings. The expression level of each gene was normalized to that of $\beta$-actin using the relative comparative CT $\left(\Delta \Delta C_{\mathrm{T}}\right)$ method. Primers and probes for this enzyme and AGT were prepared using TaqManGene Expression Assays (Applied Biosystems).

\section{Statistical Analyses}

Data are reported as mean \pm SEM. Comparisons among groups were performed using the Mann-Whitney $U$-test and a probability $(P)$ value of $<0.05$ was considered statistically significant.

\section{ACKNOWLEDGMENTS}

The authors thank Yumiko Sakurai, of the Department of Pediatrics and Adolescent Medicine, Juntendo University; Tomomi Ikeda; and Takako Ikegami of the Division of Molecular and Biochemical Research, Biomedical Research Center, Juntendo University Graduate School of Medicine; Yuuko Kojima and Shinji Nakamura, of the Division of Biomedical Imaging Research, Juntendo University Graduate School of Medicine, for their extended technical support; and Takeshi Asakura and Enago (www.enago.jp) for the English language review for detailed and precise advice related to the writing of this article.

\section{STATEMENT OF FINANCIAL SUPPORT}

No financial assistance was received to support this study.

Disclosure: The authors declare that they have no relationships that present a potential conflict of interest.

\section{REFERENCES}

1. Gluckman PD, Hanson MA. Living with the past: evolution, development, and patterns of disease. Science 2004;305:1733-6.

2. Luyckx VA, Brenner BM. Low birth weight, nephron number, and kidney disease. Kidney Int 2005;68:S68-77.

3. Lim K, Lombardo P, Schneider-Kolsky M, Hilliard L, Denton KM, Black MJ. Induction of hyperglycemia in adult intrauterine growth-restricted rats: effects on renal function. Am J Physiol Renal Physiol 2011;301: F288-94.

4. Shen Q, Xu H, Wei LM, Chen J, Liu HM, Guo W. A comparative proteomic study of nephrogenesis in intrauterine growth restriction. Pediatr Nephrol 2010;25:1063-72.

5. Shen $\mathrm{Q}, \mathrm{Xu} \mathrm{H}$, Wei LM, Chen J, Liu HM. Intrauterine growth restriction and postnatal high-protein diet affect the kidneys in adult rats. Nutrition 2011;27:364-71.

6. Neitzke U, Harder T, Plagemann A. Intrauterine growth restriction and developmental programming of the metabolic syndrome: a critical appraisal. Microcirculation 2011;18:304-11.

7. Kusuda S, Fujimura M, Uchiyama A, Totsu S, Matsunami K; Neonatal Research Network, Japan. Trends in morbidity and mortality among very-low-birth-weight infants from 2003 to 2008 in Japan. Pediatr Res 2012;72:531-8.

8. Carmody JB, Charlton JR. Short-term gestation, long-term risk: prematurity and chronic kidney disease. Pediatrics 2013;131:1168-79.

9. Taranta-Janusz K, Wasilewska A, Dębek W, Fiłonowicz R, Michaluk-Skutnik J. Urinary angiotensinogen as a novel marker of obstructive nephropathy in children. Acta Paediatr 2013;102:e429-33.

10. Saito T, Urushihara M, Kotani Y, Kagami S, Kobori H. Increased urinary angiotensinogen is precedent to increased urinary albumin in patients with type 1 diabetes. Am J Med Sci 2009;338:478-80.

11. Kobori H, Katsurada A, Ozawa Y, et al. Enhanced intrarenal oxidative stress and angiotensinogen in IgA nephropathy patients. Biochem Biophys Res Commun 2007;358:156-63.

12. Konishi Y, Nishiyama A, Morikawa T, et al. Relationship between urinary angiotensinogen and salt sensitivity of blood pressure in patients with $\operatorname{IgA}$ nephropathy. Hypertension 2011;58:205-11.

13. Nishizaki N, Hirano D, Nishizaki Y, et al. Increased urinary angiotensinogen is an effective marker of chronic renal impairment in very low birth weight children. Clin Exp Nephrol 2014;18:642-8.

14. Woods LL, Ingelfinger JR, Nyengaard JR, Rasch R. Maternal protein restriction suppresses the newborn renin-angiotensin system and programs adult hypertension in rats. Pediatr Res 2001;49:460-7.

15. Kamiyama M, Zsombok A, Kobori H. Urinary angiotensinogen as a novel early biomarker of intrarenal renin-angiotensin system activation in experimental type 1 diabetes. J Pharmacol Sci 2012;119:314-23.

16. Urushihara M, Kobori H. Angiotensinogen expression is enhanced in the progression of glomerular disease. Int J Clin Med 2011;2:378-87.

17. Dillion JJ. Angiotensinogen-converting enzyme inhibitors and angiotensinogen receptor blockers for IgA nephropathy. Semin Nephrol 2004:24:218-24.

18. Fujihara CK, Malheiros DM, Zatz R, Noronha IL. Mycophenolate mofetil attenuates renal injury in the rat remnant kidney. Kidney Int 1998;54: 1510-9.

19. Fujihara CK, Velho M, Malheiros DM, Zatz R. An extremely high dose of losartan affords superior renoprotection in the remnant model. Kidney Int 2005;67:1913-24.

20. Kobori H, Ozawa Y, Suzaki Y, Nishiyama A. Enhanced intrarenal angiotensinogen contributes to early renal injury in spontaneously hypertensive rats. J Am Soc Nephrol 2005;16:2073-80.

21. Hodgin JB, Rasoulpour M, Markowitz GS, D’Agati VD. Very low birth weight is a risk factor for secondary focal segmental glomerulosclerosis. Clin J Am Soc Nephrol 2009;4:71-6.

22. Brenner BM, Lawler EV, Mackenzie HS. The hyperfiltration theory: a paradigm shift in nephrology. Kidney Int 1996;49:1774-7.

23. Lane PH, Steffes MW, Mauer SM. Estimation of glomerular volume: a comparison of four methods. Kidney Int 1992;41:1085-9.

24. Yoshida Y, Fogo A, Ichikawa I. Glomerular hemodynamic changes vs. hypertrophy in experimental glomerular sclerosis. Kidney Int 1989;35:654-60. 